\title{
BMP9 inhibits the growth of breast cancer cells by downregulation of the PI3K/Akt signaling pathway
}

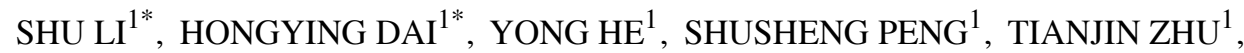 \\ YANG WU ${ }^{1}$, CHENWEI $\mathrm{LI}^{2}$ and $\mathrm{KE} \mathrm{WANG}^{1}$ \\ ${ }^{1}$ Clinical Laboratory, Yongchuan Hospital of Chongqing Medical University, Chongqing 402160; \\ ${ }^{2}$ Yubei District People's Hospital of Chongqing, Chongqing 401120, P.R. China
}

Received February 6, 2018; Accepted July 9, 2018

DOI: $10.3892 /$ or.2018.6572

\begin{abstract}
Bone morphogenetic protein 9 (BMP9) is a member of the BMP family, which is involved in the regulation of tumor biogenesis, development and metastasis. The present study aimed to investigate whether BMP9 inhibits the growth of MDA-MB-231 breast cancer cells via the phosphoinositide 3-kinase (PI3K)/Akt signaling pathway. It was shown that the expression level of BMP9 was significantly decreased, while that of phosphorylated Akt (p-Akt) was markedly increased in breast cancer tissues compared with these levels in the normal adjacent tissues. An adenovirus overexpressing BMP9 was used to infect the MDA-MB-231 cells. The expression level of p-Akt in the MDA-MB-231/BMP9 group was shown to be significantly lower than that in the MDA-MB-231/green fluorescent protein (GFP) and MDA-MB-231 control groups. The expression levels of cyclins D1, B1 and E1, c-Myc and matrix metalloproteinase 9 (MMP9) in the MDA-MB-231/BMP9 group were also reduced. The generation of a nude mouse xenograft tumor model revealed that the tumor volumes of the MDA-MB-231/BMP9 group $\left(0.32 \pm 0.05 \mathrm{~cm}^{3}\right)$ was significantly lower compared with that of the MDA-MB-231/GFP $\left(1.10 \pm 0.05 \mathrm{~cm}^{3}\right)$ and MDA-MB-231 $\left(1.12 \pm 0.12 \mathrm{~cm}^{3}\right)$ groups, and the expression level of p-Akt protein in the MDA-MB-231/BMP9 group was significantly lower compared with that of the MDA-MB-231/GFP and MDA-MB-231 groups in the nude mouse xenograft model. Taken together, these results indicate that BMP9 inhibits the growth of MDA-MB-231 breast cancer cells by inhibiting the PI3K/Akt signaling pathway both in vivo and in vitro.
\end{abstract}

Correspondence to: Dr Ke Wang, Clinical Laboratory, Yongchuan Hospital of Chongqing Medical University, Chongqing 402160, P.R. China

E-mail:wk125@126.com

${ }^{*}$ Contributed equally

Key words: bone morphogenetic protein 9, breast cancer, growth, PI3K/Akt signaling, MDA-MB-231 cells

\section{Introduction}

Breast cancer is the most common malignant tumor of women that originates in breast epithelial tissue, and it is found in women aged 40-60 years either before or after menopause. The incidence of breast cancer has been increasing year by year in recent years (1), and it has become the second most common cancer worldwide. Breast cancer accounts for $\sim 25 \%$ of all cancer-associated mortalities (2). Triple-negative breast cancer (TNBC) accounts for $\sim 12-20 \%$ of all incidences of breast cancer and is associated with early recurrence and poor prognosis $(2,3)$. TNBC is a tumor that lacks the expression of the three common cell surface receptors-estrogen receptor (ER), progesterone receptor (PR) and human epidermal growth factor receptor-2 (HER2) $(3,4)$. Therefore, TNBC evades effective treatment as far as the majority of the available targeted therapies are concerned (4). Owing to the high degree of malignancy, invasive ability and the risk of distant metastasis, TNBC has become a major focus of current research. As a representative cell line of TNBC, MDA-MB-231 cells have been extensively studied, achieving some meaningful results (5).

The family of bone morphogenetic proteins (BMPs) belongs to the transforming growth factor- $\beta$ (TGF- $\beta$ ) superfamily (6). BMPs were originally viewed as being osteoinductive cytokines that promote bone and cartilage formation in vivo (7). Recently, BMPs have been shown to be involved in the regulation of tumorigenesis, development and bone metastases (8). BMP9 [also known as growth differentiation factor 2 (GDF2)] is an integral member of the BMP family that performs different functions in different tumor types (9). For example, BMP9 may promote the proliferation and migration of liver cancer cells (10), although it inhibits the growth of osteosarcoma cells (11). Our previous study revealed that BMP9 could inhibit the invasion, migration and bone metastasis of MDA-MB-231 cells via the classical $\mathrm{BMP} / \mathrm{SMAD}$ signaling pathway (12). Upon performing further studies, we discovered that BMP9 may also have a role in other non-SMAD-dependent signaling pathways that are involved in breast cancer, including the phosphoinositide 3-kinase (PI3K)/Akt signaling pathway.

The PI3K/Akt pathway is one of the predominant pathways that are involved in the malignant progression of various 
types of tumors and mediates the proliferation, migration and invasion of breast cancer tumors. The overexpression of BMP9 reduces phosphorylation of Akt, whereas interfering with BMP9 expression increases phosphorylation of Akt (13). Furthermore, the aberrant activation of Akt is an important component in the recurrence and metastasis of tumors (14-16). Certain BMPs are able to activate the PI3K/Akt signaling pathway. Kang et al (17) identified that BMP2 may promote the invasion and migration of gastric cancer cells by activating the PI3K/Akt pathway and by upregulating matrix metalloproteinase 2 (MMP2). BMPs 4 and 7 were found to inhibit the apoptosis of ovarian granulosa cells via the PI3K/phosphoinositide-dependent kinase 1 (PDK-1)/Akt and PI3K/PDK-1/protein kinase C (PKC) signaling pathways, respectively (18). A previous study published by our research group revealed that BMP9 was able to inhibit the growth, invasion and migration of MDA-MB-231 cells (19). However, whether the inhibitory mechanism is associated with the PI3K/Akt signaling pathway has yet to be elucidated. Therefore, the present study aimed to investigate the effect of BMP9 on the proliferation of breast cancer cells and to explore the possible mechanism to potentially provide a basis for targeted therapy of breast cancer.

\section{Materials and methods}

Cell lines and adenoviral vectors. MDA-MB-231 breast cancer cells were purchased from the Cell Resource Center, Shanghai Institute for Biological Sciences (Chinese Academy of Sciences). The adenovirus that overexpresses BMP9 and the adenovirus that expresses green fluorescent protein (GFP), were obtained from the Molecular Medical Laboratory of Yongchuan Hospital of Chongqing Medical University, Chongqing, China.

Reagents. TRIzol ${ }^{\circledR}$ reagent was purchased from Takara Biotechnology Co., Ltd. (Dalian, China); L15 medium was purchased from Life Technologies (Thermo Fischer Scientific, Inc., Waltham, MA, USA). Cell protein extraction and western blot detection reagents RIPA lysis and extraction buffer were purchased from Beyotime Biotechnology Co., Ltd. (Shanghai, China). Enhanced chemiluminescence (ECL) detection kit Enlight ${ }^{\mathrm{TM}}$ was purchased from Engreen Biological Co., Ltd. (Beijing, China). Phosphorylase and protease inhibitor cocktail tablets PhosSTOP were purchased from Roche Diagnostics (Basel, Switzerland). Mouse monoclonal GAPDH antibody (cat. no. 97166), Akt total protein mouse monoclonal (cat. no. 2920) and Akt phosphoprotein rabbit monoclonal (specific to Ser473; cat. no. 4060) antibodies, cyclin E1 monoclonal antibody (cat. no. 4129), cyclin D1 rabbit monoclonal antibody (cat. no. 2978) and cyclin B1 mouse monoclonal antibody (cat. no. 4135) were purchased from Cell Signaling Technology, Inc. (Danvers, MA, USA). MM9 mouse monoclonal antibody (cat. no. ab58803) and BMP9 rabbit polyclonal antibody (cat. no. ab71809) were obtained from Abcam (Cambridge MA, USA). PV9001 horseradish enzyme anti-rabbit immunoglobulin G (IgG) polymer, PV9002 horseradish enzyme anti-mouse $\mathrm{IgG}$ polymer, and the DAB display reagent were purchased from Zhongshan Golden Bridge Biological Co., Ltd. (Beijing, China).
Patients and animals. A total of 23 pairs of breast cancer and adjacent normal tissues (23 females, aged 25-77 years) were obtained from the subjects (Table I), which were confirmed by pathological examination using $\mathrm{H} \& \mathrm{E}$ staining. All samples were collected from the Yongchuan Hospital of Chongqing Medical University from May 2016 to August 2017. All the experiments concerning the tumor specimens were approved by the Ethics Association of Yongchuan Hospital of the Chongqing Medical University, and all the patients signed the informed consent form.

Four-week-old male nude mice (weight $21 \mathrm{~g})(\mathrm{BALB} / \mathrm{c}$; Beijing Hua Fukang Biological Polytron Technologies Inc., Beijing, China) were used for generation of the xenograft model. All experiments were approved by the Institutional Animal Care and Use Committee of the Chongqing Medical University, as well as regional authorities [reference number, SYXK(YU)2014-0001] in accordance with the 'Guidelines for the Welfare of Animals in Experimental Neoplasia' (The China Coordinating Committee on Cancer Research). The animals were housed in individually ventilated cages under sterile conditions and were granted unrestricted access to food and water. At the end of the experimental period, the animals were sacrificed by excessive ether inhalation.

Immunohistochemistry. The prepared tissue sections were dewaxed, hydrated and antigen retrieval was performed. After washing three times with PBS (5 min each wash), antibodies against BMP9, Akt and phosphorylated Akt were added dropwise followed by incubation in the humid chamber at $37^{\circ} \mathrm{C}$ for $2 \mathrm{~h}$. Following three further washes with PBS (5 min each wash), a secondary antibody (PV9001 or PV9002) was added, and the sections were incubated at $37^{\circ} \mathrm{C}$ for a further $20 \mathrm{~min}$. DAB and hematoxylin counterstain were added for $3 \mathrm{~min}$ followed by routine dehydration to maintain transparency and sealing. Each slice was photographed using the BX51P Olypmus polarizing microscope (Olympus Corp., Tokyo, Japan) at a low magnification x100 (with 10 randomly selected fields of view), and the cumulative optical density of the dyed yellow areas was assessed using ImageJ software (National Institutes of Health, Bethesda, MD, USA).

Cell culture and infection. MDA-MB-231 cells were cultured in L15 culture medium containing 10\% fetal bovine serum(FBS) (including $1 \%$ penicillin and streptomycin) at $37^{\circ} \mathrm{C}$, in the absence of $\mathrm{CO}_{2}$. When the cells had reached a confluency of $\sim 70-80 \%$, they were digested and passaged with $0.25 \%$ trypsin (containing $0.02 \%$ EDTA $2 \mathrm{Na}$ ), and the culture was continued. MDA-MB-231 cells in the exponential phase of growth were seeded at a density of $2 \times 10^{6}$ culture flask. After $24 \mathrm{~h}$, the cells were subsequently infected with adenovirus vehicles that express GFP and recombinant BMP-9. After a further 8-12 h cultivation, the medium was replaced by fresh medium, and the fluorescence was subsequently observed $24 \mathrm{~h}$ later using a fluorescence microscope (Olympus Imaging IX71; Olympus Corp.).

Real-time quantitative polymerase chain reaction ( $R T-q P C R)$. The total RNA of the MDA-MB-231 cells, and the total RNA of the MDA-MB-231/BMP9 and MDA-MB-231/GFP cells were extracted using the TRIzol ${ }^{\circledR}$ method after $24 \mathrm{~h}$ 
Table I. Clinicopathological features of the 23 breast cancer patients recruited to the study.

\begin{tabular}{ccccccc}
\hline Patient & Age (years) & Sex & Tumor grade & Tumor stage & Lymph node metastasis & Tumor size (cm) \\
\hline 1 & 51 & Female & II & T2N3M0 & Yes & $2.5 \times 2 \times 1$ \\
2 & 53 & Female & I & T1N1M0 & Yes & $1.5 \times 1.5 \times 1$ \\
3 & 47 & Female & II & T1N0M0 & No & $2 \times 2 \times 1$ \\
4 & 54 & Female & II & T1N1M0 & Yes & $3 \times 2 \times 1$ \\
5 & 53 & Female & II & T1N1M0 & Yes & $1.8 \times 1.5 \times 1$ \\
6 & 49 & Female & II & T1N1M0 & Yes & $1.5 \times 1 \times 1$ \\
7 & 45 & Female & III & T1N1M0 & Yes & $2.5 \times 1 \times 1.5$ \\
8 & 64 & Female & II & T2N0M0 & No & $2 \times 1.8 \times 1.5$ \\
9 & 37 & Female & II & T1N0M0 & No & $1.5 \times 1.2 \times 1$ \\
10 & 53 & Female & II & T1N0M0 & No & $1 \times 1 \times 1$ \\
11 & 52 & Female & II & T1N0M0 & No & $0.8 \times 0.8 \times 0.7$ \\
12 & 50 & Female & II & T2N0M0 & No & $2.5 \times 2 \times 1.8$ \\
13 & 55 & Female & II & T1N0M0 & No & $1.5 \times 1.3 \times 1$ \\
14 & 74 & Female & II & T1N1M0 & Yes & $1.5 \times 1.5 \times 1.2$ \\
15 & 53 & Female & II & T1N0M0 & No & $2 \times 1.5 \times 1$ \\
16 & 58 & Female & II & T1N0M0 & No & $0.8 \times 0.8 \times 0.6$ \\
17 & 25 & Female & II & T2N1M0 & Yes & $2.5 \times 1.5 \times 1$ \\
18 & 43 & Female & III & T1N0M0 & Yes & $2 \times 2 \times 1.2$ \\
19 & 53 & Female & II & T1N0M0 & Yes & $1 \times 0.8 \times 0.8$ \\
20 & 43 & Female & II & T2N0M0 & Yes & $2.5 \times 1.8 \times 1.6$ \\
21 & 77 & Female & II & T1N0M0 & No & $2 \times 1.6 \times 1.5$ \\
22 & 50 & Female & II & T1N0M0 & Yes & $2 \times 1.8 \times 1.5$ \\
23 & 42 & Female & I & T2N3M0 & No & $3 \times 2.5 \times 1.2$ \\
\hline
\end{tabular}

of adenovirus infection (12). Aliquots $(2 \mu \mathrm{g})$ of total RNA were used to synthesize cDNA using a reverse transcription kit, and subsequently $1 \mu \mathrm{l}$ cDNA was used as a template for PCR amplification. The following primer sequences were used: GAPDH, sense, 5'-CAGCGACACCCACTCCTC-3' and antisense, 5'-TGAGGTCCACCACCCTGT-3'; cyclin B1 sense, 5'-GCACTTTCCTCCTTCTCA-3' and antisense, 5'-CGA TGTGGCATACTTGTT-3'; cyclin D1 sense, 5'-GCGAGG AACAGAAGTGCG-3' and antisense, 5'-GGAGTTGTCGGT GTAGATGC-3'; cyclin E1 sense, 5'-TGGATGTTGACTGCC TTGA-3' and antisense, 5'-GTCGCACCACTGATACCCT-3'; c-Myc sense, 5'-ACACCCTTCTCCCTTCG-3' and antisense, 5'-CCGCTCCACATACAGTCC-3'; and MMP9 sense, 5'-GAA CTTTGACAGCGACAAGA-3' and antisense, 5'-GGCGAG GACCATAGAGG-3'. The PCR reaction conditions were as follows: An initial step at $95^{\circ} \mathrm{C}$ for $30 \mathrm{sec}$; denaturation at $95^{\circ} \mathrm{C}$ for $5 \mathrm{sec}$; followed by annealing for $30 \mathrm{sec}$ at $55^{\circ} \mathrm{C}$ and extension for $10 \mathrm{sec}$ at $72^{\circ} \mathrm{C}$ in a total of 40 cycles. The experiment was repeated three times. The $2^{-\Delta \Delta \mathrm{Cq}}$ method was utilized to normalize the expression of target mRNAs relative to the reference gene, GAPDH (12).

Western blotting. A density of 5x10 $10^{5} \mathrm{MDA}-\mathrm{MB}-231$ cells/culture flask were passaged into $6-\mathrm{cm}$ diameter Petri dishes. When the cell confluency reached $\sim 50-60 \%$, the adenovirus that overexpresses BMP9 was added, and the medium was subsequently replaced with serum-free L15 medium after $24 \mathrm{~h}$. The cells were cultivated for a further $48 \mathrm{~h}$, at which time the culture solution was discarded, and the cells were subsequently washed twice with pre-cooled PBS. Cell lysate (150 $\mu \mathrm{l} /$ Petri dish) was added, followed by lysis for $30 \mathrm{~min}$ on ice, and the cells were centrifuged at $4^{\circ} \mathrm{C}$ for $15 \mathrm{~min}$ at $12,000 \mathrm{rpm}(10,000 \mathrm{x} \mathrm{g} / \mathrm{min})$. The supernatant was then transferred to a $1.5 \mathrm{ml} \mathrm{EP}$ tube, prior to utilizing the bicinchoninic acid (BCA) method for the determination of protein concentration. Aliquots $(15 \mu \mathrm{l})$ of the protein solution were extracted for protein electrophoresis (SDS-PAGE; concentration of gel 5\%, separation gel, $10 \%)$. The proteins were electrotransferred onto a polyvinylidene difluoride (PVDF) membrane, and then blocked with 5\% bovine serum albumin (BSA) at $37^{\circ} \mathrm{C}$ for $1 \mathrm{~h}$. The primary antibodies against GAPDH (dilution 1:1,000), Akt (dilution 1:1,000), phosphorylated Akt (p-Akt; dilution 1:1,000), c-Myc (dilution 1:1,000), cyclin D1 (dilution 1:1,000), cyclin B1 (dilution 1:1,000), cyclin E1 (dilution 1:1,000) and MMP9 (dilution 1:500) were added and incubated overnight at $4^{\circ} \mathrm{C}$. The membranes were then washed three times ( $15 \mathrm{~min}$ each wash) in $0.1 \%$ Tris-buffered saline/Tween (TBST) solution, after which the corresponding secondary antibody (goat anti-rabbit or goat anti-mouse) (dilution, 1:5,000) was added. The cells were incubated for $1 \mathrm{~h}$ at $37^{\circ} \mathrm{C}$, and the PVDF membranes were washed three times (15 min each wash) in $0.1 \%$ TBST solution. Finally, the ECL chromogenic solution was used for imaging. GEL EQ (Bio-Rad Laboratories, Hercules, CA, USA) was used in the gel imager, and Quantity One ${ }^{\circledR}$ software (Bio-Rad Laboratories, Inc.) was used to analyze the relative optical densities. The relative expression level of the target 
gene protein was calculated according to the gray value of the target gene band/the gray value of the internal reference band.

Animal models. Trypsin solution $(0.25 \%)$ was used to digest the MDA-MB-231 cells, and the MDA-MB-231/GFP and MDA-MB-231/BMP9 cells in the exponential growth phase $24 \mathrm{~h}$ following adenovirus infection. Tumor cells were injected subcutaneously into the backs of the nude mice for each group of five nude mice $(0.1 \mathrm{ml} /$ nude mouse; adjusted cell density, $5 \times 10^{6}$ cells $\left./ \mathrm{ml}\right)$. Once the tumors of any group were visible and palpable, the starting point measurements were taken. The diameters of the tumor were subsequently recorded every three days. The volume (V) was calculated based on the following formula: $V=4 / 3 \pi \mathrm{l} / 2 \mathrm{w} / 2 \mathrm{~h} / 2$, (where $\pi=3.14$, w is the width, $l$ is the length and $h$ is the height), and the growth curves of the xenografts were plotted. Following inoculation of the tumor cells, all nude mice were sacrificed on the 25th day after the animal model had been successfully constructed. The tumor tissue was dissected, and the tumor size was measured. The transplanted tumors were subsequently stripped, fixed in $4 \%$ polyformaldehyde solution, paraffin-embedded, and then sectioned. The tumor tissue sections were stained with H\&E prior to immunohistochemistry and analysis using Image J software (National Institutes of Health).

Statistical analysis. SPSS version 20.0 statistical software (IBM Corp., Armonk, NY, USA) was used to analyze the experimental data. The results are expressed as the mean \pm standard deviation (SD). The expression of BMP9 or p-AKT using immunohistochemical staining in breast cancer specimens was analyzed by paired Student's t-test. The correlation between BMP9 and p-Akt was analyzed by the Spearman correlation coefficient method. The other data were analyzed by analysis of variance (ANOVA). Multiple comparison between the groups was performed using the S-N-K method. The expression levels of cyclin D1, cyclin B1, cyclin E1, c-Myc and MMP9 were analyzed using RT-qPCR and the $2^{-\Delta \Delta \mathrm{Cq}}$ method. $\mathrm{P}<0.05$ was considered to indicate a statistically significant difference.

\section{Results}

Expression level of BMP9 is downregulated in breast cancer tissues, whereas the expression of p-Akt is upregulated. In the 23 clinical breast cancer specimens, immunohistochemical staining showed that the positive rate of BMP9 was $17.4 \%(4 / 23)$ in the tumor tissues, whereas the positive rate of BMP9 was $65.2 \%(15 / 23)$ in the paracancerous tissues. The expression level of BMP9 was significantly lower in the tumor tissues compared with the level noted in the paracancerous tissues $(\mathrm{P}<0.05)$. The positive rate of $\mathrm{p}$-Akt was $82.6 \%(19 / 23)$ in the cancer tissues, whereas the positive rate of $\mathrm{p}-\mathrm{Akt}$ was $47.8 \%(11 / 23)$ in the paracancerous tissues. The protein expression level of $\mathrm{p}$-Akt was significantly higher $(\mathrm{P}<0.05)$ in cancer tissues than the level noted in the paracancerous tissues (Fig. 1A and B). Spearman correlation coefficient analysis revealed that the expression level of BMP9 was negatively correlated with the $\mathrm{p}$-Akt protein expression level in tumor tissue $(\mathrm{r}=-0.422 ; \mathrm{P}<0.05)$ (Fig. 1C). These results supported that the PI3K/Akt signaling pathway is involved in the development and progression of breast cancer, since the expression level of BMP9 was negatively correlated with the expression level of p-Akt.

BMP9 inhibits the PI3K/Akt signaling pathway and downregulates the expression levels of cyclin D1, cyclin $B 1$, cyclin E1, c-Myc and MMP9 in MDA-MB-231 cells. To investigate whether BMP9 exerts an inhibitory effect on the PI3K/Akt signaling pathway, western blotting was performed. Western blotting revealed that the protein expression level of p-Akt in the BMP9 overexpression group (MDA-MB-231/BMP9) was significantly lower compared with the GFP group (MDA-MB-231/GFP) and the blank control group (MDA-MB-231) after the MDA-MB-231 cells had been infected with the adenovirus overexpressing BMP9 for $48 \mathrm{~h}$. These results suggested that BMP9 inhibited the PI3K/Akt signaling pathway.

Cyclins D1, B1 and E1, c-Myc, and MMP9 have been reported to be involved in the proliferation and invasion of tumor cells (20-24). Therefore, RT-qPCR was used to estimate their expression levels in recombinant MDA-MB-231/BMP9 cells. The expression levels of cyclin D1, cyclin B1, cyclin E1, c-Myc and MMP9 were all shown to be downregulated in the MDA-MB-231/BMP9 cells (cyclin D1: $2^{-\Delta \Delta \mathrm{Cq}}=0.321$; cyclin B1: $2^{-\Delta \Delta \mathrm{Cq}}=0.287$; cyclin $\mathrm{E} 1: 2^{-\Delta \Delta \mathrm{Cq}}=0.277$; c-Myc: $2^{-\Delta \Delta \mathrm{Cq}}=0.586$; MMP9: $2^{-\Delta \Delta \mathrm{Cq}}=0.130$ ) (Fig. 2A). Western blotting was performed to confirm the expression levels of cyclin D1, cyclin B1, cyclin E1, c-Myc and MMP9 in the MDA-MB-231/BMP9 groups. The results demonstrated that these proteins were significantly lower in MDA-MB-231/BMP9 cells compared with these level in the MDA-MB-231/GFP and MDA-MB-231 groups (Fig. 2B). These results suggested that BMP9 inhibited the proliferation of MDA-MB-231 cells via downregulation of the expression levels of cyclin D1, cyclin B1, cyclin E1, c-Myc and MMP9.

Overexpression of BMP9 inhibits the growth of breast cancer tumors in a nude mouse xenograft tumor model. After the successful generation of the nude mouse xenograft tumor model, tumors were identified on the 10th day in the MDA-MB-231/GFP and the MDA-MB-231 groups, although tumors were found on the 16th day in the MDA-MB-231/BMP9 group. All the nude mice were sacrificed on the 25th day after the animal model had been successfully constructed, and the tumor tissue was dissected. The size of the tumors was measured using a Vernier caliper (the maximum tumour diameter: MDA-MB-231/BMP9 group was $1.08 \mathrm{~cm}$; MDA-MB-231/GFP group was $1.48 \mathrm{~cm}$; MDA-MB-231 group was $1.49 \mathrm{~cm}$ on the 25 th day). The tumor volume in the MDA-MB-231/BMP9 group $\left(0.32 \pm 0.05 \mathrm{~cm}^{3}\right)$ was markedly lower when compared with that in the MDA-MB-231/GFP group $\left(1.10 \pm 0.05 \mathrm{~cm}^{3}\right)$ and MDA-MB-231 group $\left(1.12 \pm 0.12 \mathrm{~cm}^{3}\right) \quad(\mathrm{P}<0.001$; Fig. 3A and B). At the same time, the tumors of the MDA-MB-231/GFP and MDA-MB-231 groups were found to be necrotic and ulcerated when the tumors were peeled off. Owing to the adhesion between the tumor and the skin of the nude mice, the tumors were not easy to peel off. By contrast, the tumor masses of the MDA-MB-231/BMP9 group were found to be hard, there was no obvious necrosis, and the tumors were easy to peel off. These results demonstrated 

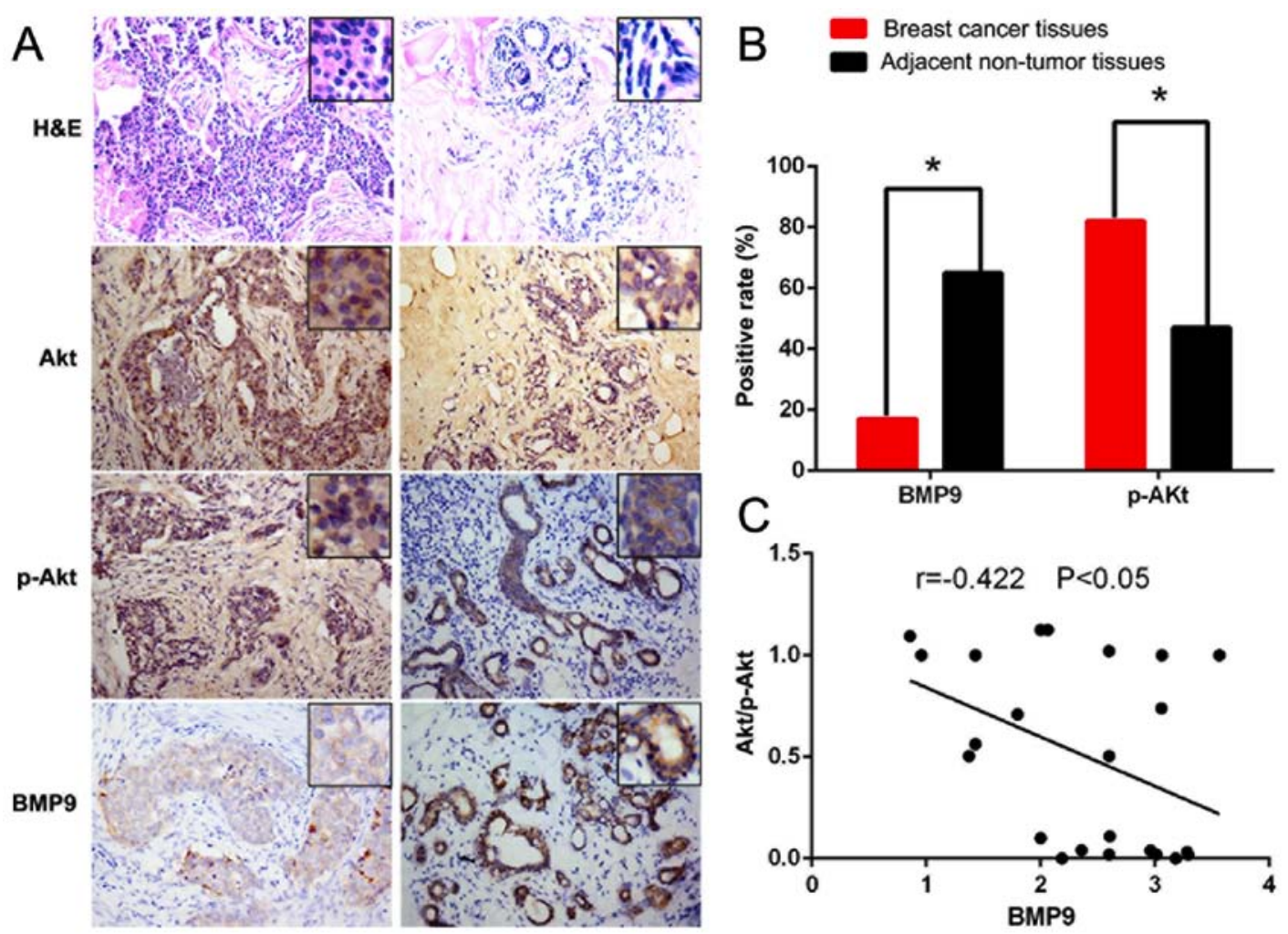

Figure 1. Expression levels of BMP9, Akt, and p-Akt in breast cancer tissues and adjacent normal tissues as determined by immunohistochemistry. (A) Immunohistochemistry was used to detect the expression of BMP9, Akt, and p-Akt in breast cancer tissues and adjacent normal tissues (magnification, x100). (B) Quantification of the positive rate of BMP9 and p-Akt in the breast cancer and adjacent non-tumor tissues. "P<0.05. (C) Correlation between p-Akt and BMP9 expression in all 23 human breast cancer tumor tissues. BMP9, bone morphogenetic protein 9; p-Akt, phosphorylated Akt.

A
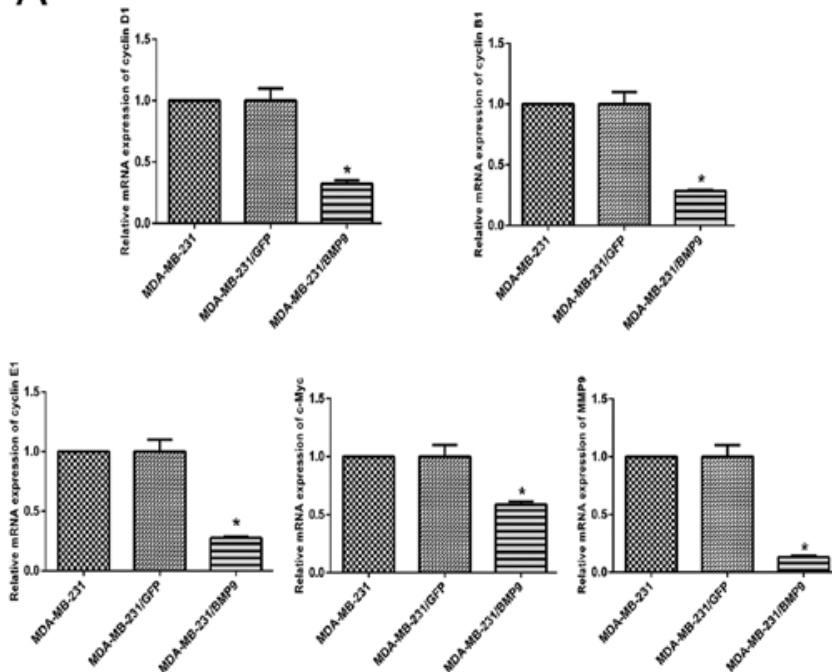

B

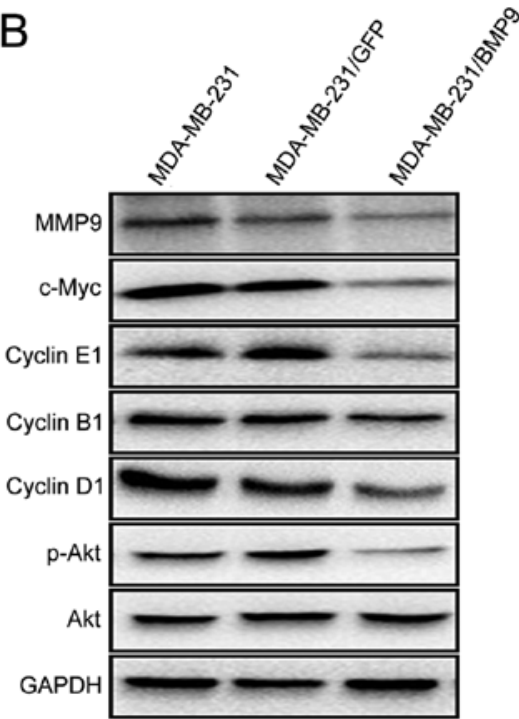

Figure 2. Expression levels of cyclin D1, cyclin B1, cyclin E1, c-Myc and MMP9 as determined by (A) RT-qPCR and (B) western blotting. (A) The mRNA expression levels of cyclin D1, cyclin B1, cyclin E1, c-Myc and MMP9 for the MDA-MB-231/BMP9 group were significantly decreased compared with the expression levels in the MDA-MB-231/GFP and MDA-MB-231 groups ( $\mathrm{P}<0.05)$. (B) The protein expression level of p-Akt was significantly lower in the MDA-MB-231/BMP9 group compared with that noted in the MDA-MB-231/GFP and MDA-MB-231 groups p-Akt, phosphorylated Akt; MMP9, matrix metalloproteinase 9; RT-qPCR, real-time quantitative polymerase chain reaction.

that the overexpression of BMP9 may considerably inhibit the growth of breast cancer cells.

BMP9 inhibits PI3K/Akt expression in the nude mouse xenograft tumor model. In the nude mouse xenograft tumor model, immunohistochemical staining revealed that p-Akt protein expression was obviously decreased in the MDA-MB-231/BMP9 group compared with that noted in the MDA-MB-231/GFP and MDA-MB-231 groups. The t-Akt protein expression exhibited no difference in the three groups. 
A

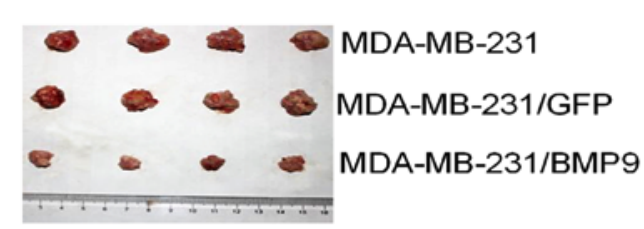

B

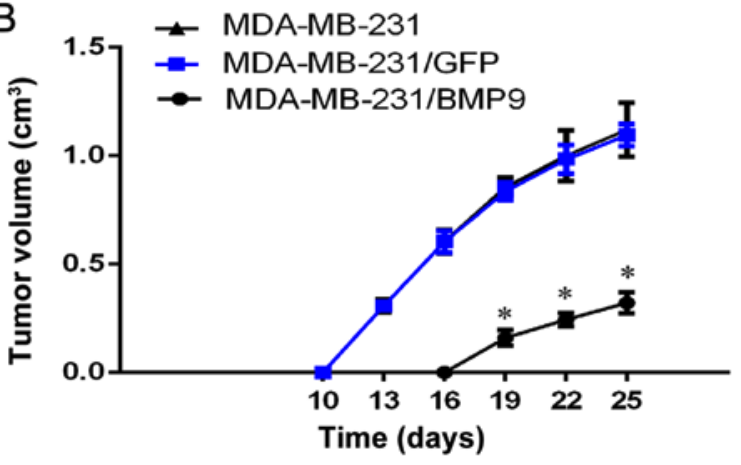

Figure 3. Effect of BMP9 on a nude mouse xenograft model. (A) The tumor volumes of xenografts formed by the MDA-MB-231/BMP9, MDA-MB-231/ GFP and MDA-MB-231 breast cancer cells were determined. (B) Growth curves of xenografts formed by MDA-MB-231/BMP9, MDA-MB-231/GFP and MDA-MB-231 breast cancer cells were plotted. "P<0.001. BMP9, bone morphogenetic protein 9; GFP, green fluorescent protein.

This result further confirmed that BMP9 inhibits the growth of breast cancer by inhibiting the PI3K/Akt signaling pathway in vivo.

\section{Discussion}

Previous studies have revealed that BMPs are able to promote bone formation, cell invasion and metastasis via the classical BMP/SMAD signaling pathway (12). Recent studies have reported the effect of BMPs on certain non-classical signal transduction pathways, including those involving PI3K/Akt and mitogen-activated protein kinases (MAPKs) (25-28). These non-classical BMP/SMAD signaling pathways have an important role in tumor growth, invasion and metastasis. Pancholi et al (29) demonstrated that the estrogen receptor (ER) could bind to both ERBB2 and PI3K, leading to the phosphorylation of Akt. Another study by Lin et al (30) confirmed that Akt is the main downstream target of PI3K, demonstrating that PI3K and Akt may work together to participate in tumorigenesis, development and metastasis. It has been reported that most BMPs can promote tumor growth, invasion and metastasis via the PI3K/Akt signaling pathway. Ren et al (13) showed that PI3K/Akt activation could promote the proliferation, migration and invasion of HER2-positive SK-BR-3 breast cancer cells, whereas Duan et al (31) showed that inhibition of the PI3K/Akt pathway could inhibit cell proliferation and migration in gastric cancer.

BMP9 was originally cloned from a fetal mouse liver cDNA. It has been shown as a pleiotropic cytokine involved in a number of physiologic events. These include hepatic reticuloendothelial system, bone morphogenesis, neuronal differentiation, hematopoiesis, angiogenesis, iron homeostasis, glucose homeostasis and cancer growth or metastasis. In our
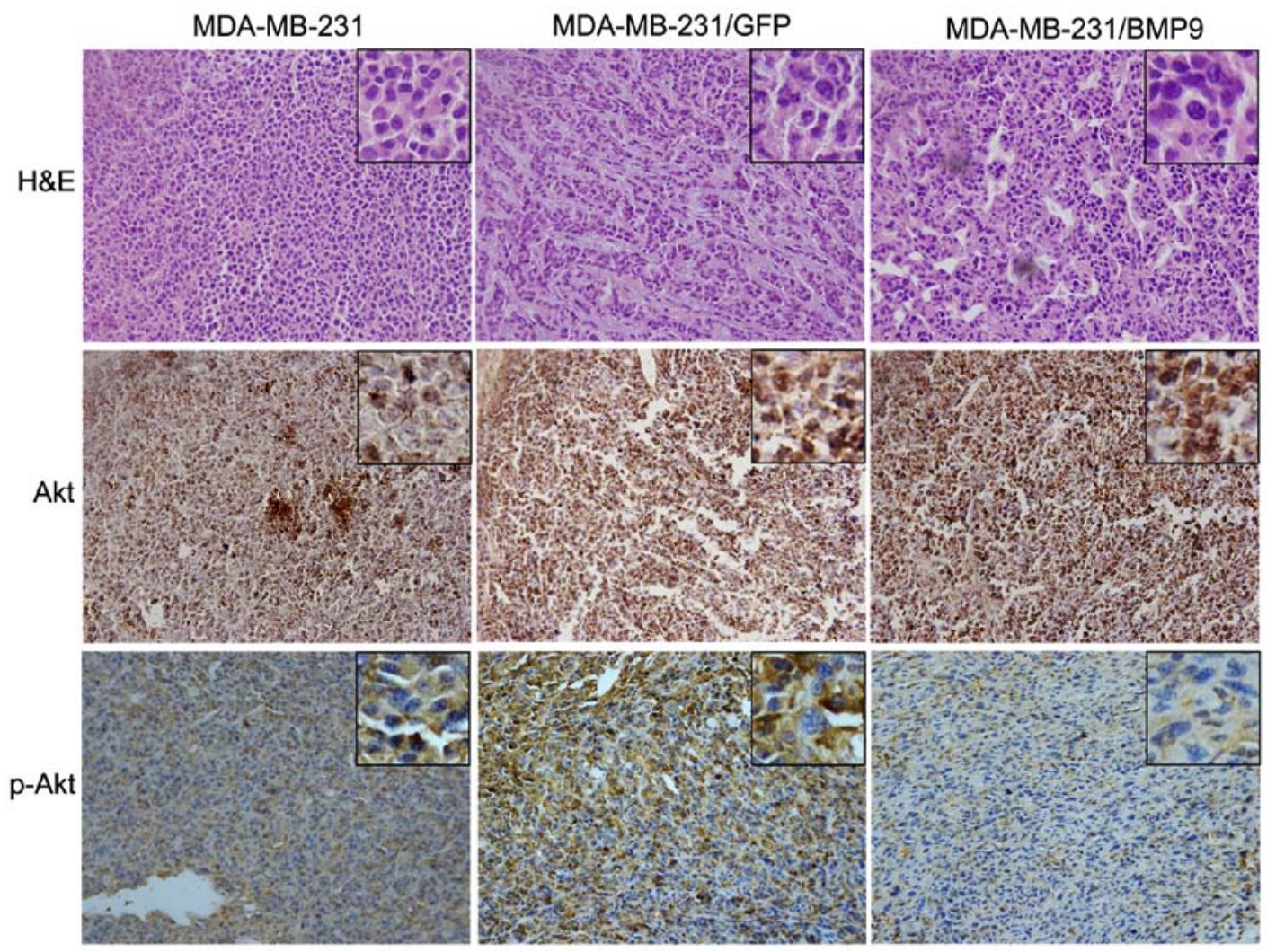

Figure 4. Immunohistochemical staining results of xenograft tumors derived from the MDA-MB-231/BMP9, MDA-MB-231/GFP and MDA-MB-231 breast cancer cells. Hematoxylin and eosin (H\&E) staining and Immunohistochemistry of the tumors in the different groups (magnification, x100). BMP9, bone morphogenetic protein 9; GFP, green fluorescent protein; p-Akt, phosphorylated Akt. 
previous studies, HCT116 cells were infected with the BMP9 adenovirus to construct conditioned medium, and we found that BMP9 mRNA was readily detectable in the HCT116/BMP9 cells, and the conditioned medium indeed contained detectable levels of BMP9 protein (9). Our research confirm a previous study by Ye et al that BMP9 was released into the medium following transfection of the gene into cells (32).

BMP9, as a multifunctional cytokine, not only induces bone and cartilage formation but is also involved in the proliferation, differentiation, invasion and metastasis of tumor cells. A previous study by our research laboratory demonstrated that BMP9 could inhibit the proliferation of lung adenocarcinoma A549 cells via the SMAD-dependent BMP/SMAD signaling pathway (33). Our previous research also revealed that BMP9 can inhibit the proliferation, invasion, and migration of breast cancer cells via the activin receptor-like kinase-2 (ALK2)-dependent BMP/SMAD signaling pathway (19). This prompted us to consider whether BMP9 exerts an effect on certain non-classical signal transduction pathways, and therefore our research studies were aimed to elucidate the mechanism of BMP9 in breast cancer cells in order to provide a theoretical basis for the targeted therapy of breast cancer.

In the present study, 23 cases of patients with clinical breast cancer were first identified. Immunohistochemical staining showed that the positive rate of BMP9 expression in breast cancer was significantly lower when compared with that in adjacent normal tissues $(\mathrm{P}<0.05)$. In addition, the protein expression level of $\mathrm{p}$-Akt was significantly reduced in the samples with BMP9 expression. Furthermore, the expression level of BMP9 in tumor tissue was negatively correlated with the expression level of $\mathrm{p}-$ Akt protein $(\mathrm{r}=-0.422 ; \mathrm{P}<0.05)$ (Fig. 1C). These preliminary results suggest that BMP9 may participate in the growth, invasion, and metastasis of breast cancer cells via the PI3K/Akt signaling pathway. Subsequently, BMP9 was overexpressed in MDA-MB-231 breast cancer cells by adenovirus infection in vitro, revealing that the overexpression of BMP9 inhibited the protein expression level of p-Akt (Fig. 2B). Experiments performed in vivo further confirmed that BMP9 serves an inhibitory role in the growth, invasion, and migration of breast cancer cells, and that a clear association exists with the PI3K/Akt signaling pathway. In addition to acting via the classical BMP/SMAD signaling pathway, BMP9 also functions through the non-SMAD-dependent PI3K/Akt signaling pathway.

To further explore the carcinogenic mechanism of BMP9 in breast cancer, a nude mouse xenograft tumor model was constructed. The animal experiments demonstrated that BMP9 can inhibit breast cancer growth (Fig. 3A and B). After constructing the nude mouse model over a 28-day period, the nude mice were sacrificed and the tumor tissues were isolated and paraffin-embedded. Immunohistochemistry results showed that the positive percentage of $\mathrm{p}$-Akt protein in the MDA-MB-231/BMP9 group was significantly lower compared with the MDA-MB-231 and MDA-MB-231/GFP groups (Fig. 4). These results suggested that BMP9 can indeed inhibit the growth of MDA-MB-231 cells by inhibiting the PI3K/Akt signaling pathway in vivo, thereby providing experimental data in support of the clinical application of BMP9 in the treatment of breast cancer.
As a multifunctional factor, the role of BMP9 in osteogenesis and breast cancer has been progressively determined. Accumulating evidence has shown that BMP9 may exert a role through multiple signaling pathways (34-36). The study has also, in part, explained why BMPs serve different roles in different tumors, and even for the same BMP in different tumors, its role may significantly differ. For example, BMP9 was found to have an inhibitory role in prostate cancer and breast cancer, whereas BMP9 had a role in promoting ovarian cancer $(32,37)$. We demonstrated that BMP9 can inhibit the PI3K/Akt signaling pathway. Yet, we did not ascertain how BMP9 regulates the PI3K/Akt signaling pathway. We will knock down the BMP9 type I receptor (ALK1 or ALK2) to inhibit the BMP9-independent SMAD signaling pathways in order to determine the effect of BMP9 overexpression on breast tumor cell growth in future studies. The published data indicate that ALK1 or ALK2 belongs to BMP9 type I receptors and can bind with BMP9. Knocking down each of the BMP9 type I receptors can directly inhibit BMP9 signaling pathways. It will confirm whether BMP9 can directly target the PI3K/AKT signaling pathway or not (13).

The present study has shown that BMP9 can inhibit the PI3K/Akt signaling pathway consequently suppressing breast cancer growth. In addition, it has been shown by RT-qPCR and western blotting that BMP9 may inhibit the expression levels of cyclin D1, cyclin B1, cyclin E1, c-Myc and MMP9 (Fig. 2A and B), which subsequently leads to inhibition of the proliferation, invasion, and metastasis of breast cancer cells. These results may explain, in part, how the degree of malignancy of MDA-MB-231 cells was increased in our previous studies after the inhibitory effect of BMP9 on connective tissue growth factor (CTGF) had been restored in MDA-MB-231 cells by using an adenovirus co-infection technique in vitro (12), although certain differences existed compared with the blank control group. Since BMP9 can exert a role via the BMP/SMAD signaling pathway as well as through the non-classical PI3K/Akt signal transduction pathway, this may also explain why BMP9 considerably inhibited the growth of MDA-MB-231 cells.

In conclusion, the present study has shown that BMP9 expression was downregulated in breast cancer cells. This study has shown, to the best of our knowledge for the first time, that BMP9 inhibits the growth of breast cancer by inhibiting the PI3K/Akt signaling pathway. At the same time, BMP9 can also inhibit the proliferation, invasion, and metastasis of breast cancer by inhibiting the expression of cyclin D1, cyclin B1, cyclin E1, c-Myc, and MMP9. Therefore, BMP9-mediated PI3K/Akt signaling may serve as a novel target for potential breast cancer therapy to prevent proliferation, invasion, and metastasis of breast cancer cells.

\section{Acknowledgements}

Not applicable.

\section{Funding}

The present study was supported by the Natural Science Foundation Project of Yongchuan (grant nos. Ycstc.2016nc510 and Ycstc.2017nc5020), the Natural Science Foundation Project 
of Chongqing Education Committee (grant no. KJ1500202) and the Natural Science Foundation Project of Yongchuan Hospital (grant no. YJZQN201517).

\section{Availability of data and material}

The datasets used and/or analysed during the current study are available from the corresponding author on reasonable request.

\section{Authors' contributions}

HD, SL, TZ, YW, CL and SP performed the research. KW and SL designed the research study. SL, YH and HD analyzed the data. SL wrote the paper. KW, as the corresponding author, modified the article and proposed its written content. All authors have read and approved the final version of the manuscript and agree to be accounTable for all aspects of the research in ensuring that the accuracy or integrity of any part of the work are appropriately investigated and resolved.

\section{Ethics approval and consent to participate}

All the experiments concerning the tumor specimens were approved by the Ethics Association of Yongchuan Hospital of the Chongqing Medical University, and all the patients signed the informed consent form. All experiments were approved by the Institutional Animal Care and Use Committee of the Chongqing Medical University, as well as regional authorities [reference no. SYXK(YU)2014-0001] in accordance with the 'Guidelines for the Welfare of Animals in Experimental Neoplasia' (The China Coordinating Committee on Cancer Research).

\section{Patient consent for publication}

All the patients signed the informed consent form.

\section{Competing interests}

The authors state that they have no competing interests.

\section{References}

1. Zhang Z, Ni C, Chen W, Wu P, Wang Z, Yin J, Huang J and Qiu F: Expression of CXCR4 and breast cancer prognosis: A systematic review and meta-analysis. BMC Cancer 14: 49, 2014.

2. Richards CE, Vellanki SH, Smith YE and Hopkins AM: Diterpenoid natural compound $\mathrm{C} 4$ (Crassin) exerts cytostatic effects on triple-negative breast cancer cells via a pathway involving reactive oxygen species. Cell Oncol 41: 35-46, 2018.

3. Chacón RD and Costanzo MV: Triple-negative breast cancer. Breast Cancer Res 12 (Suppl 2): S3, 2010.

4. Foulkes WD, Smith IE and Reis-Filho JS: Triple-negative breast cancer. N Engl J Med 363: 1938-1948, 2010.

5. Ma H, Xu X, Clague J, Lu Y, Togawa K, Wang SS, Clarke CA Lee E, Park HL, Sullivan-Halley J, et al: Recreational physical activity and risk of triple negative breast cancer in the California Teachers Study. Breast Cancer Res 18: 62, 2016.

6. Wang K, Feng H, Ren W, Sun X, Luo J, Tang M, Zhou L, Weng Y, He TC and Zhang Y: BMP9 inhibits the proliferation and invasiveness of breast cancer cells MDA-MB-231. J Cancer Res Clin Oncol 137: 1687-1696, 2011.

7. Wang RN, Green J, Wang Z, Deng Y, Qiao M, Peabody M, Zhang Q, Ye J, Yan Z, Denduluri S, et al: Bone morphogenetic protein (BMP) signaling in development and human diseases. Genes Dis 1: 87-105, 2014.
8. Ye L, Bokobza SM and Jiang WG: Bone morphogenetic proteins in development and progression of breast cancer and therapeutic potential (Review). Int J Mol Med 24: 591-597, 2009.

9. Wan S, Liu Y, Weng Y, Wang W, Ren W, Fei C, Chen Y, Zhang Z, Wang T, Wang J, et al: BMP9 regulates cross-talk between breast cancer cells and bone marrow-derived mesenchymal stem cells. Cell Oncol 37: 363-375, 2014.

10. García-Álvaro M, Addante A, Roncero C, Fernández M, Fabregat I, Sánchez A and Herrera B: BMP9-induced survival effect in liver tumor cells requires p38MAPK activation. Int J Mol Sci 16: 20431-20448, 2015.

11. Lv Z, Wang C, Yuan T, Liu Y, Song T, Liu Y, Chen C, Yang M, Tang $\mathrm{Z}$, Shi $\mathrm{Q}$, et al: Bone morphogenetic protein 9 regulates tumor growth of osteosarcoma cells through the Wnt/ $\beta$-catenin pathway. Oncol Rep 31: 989-994, 2014.

12. Ren W, Sun X, Wang K, Feng H, Liu Y, Fei C, Wan S, Wang W, Luo J, Shi Q, et al: BMP9 inhibits the bone metastasis of breast cancer cells by downregulating CCN2 (connective tissue growth factor, CTGF) expression. Mol Biol Rep 41: 1373-1383, 2014.

13. Ren W, Liu Y, Wan S, Fei C, Wang W, Chen Y, Zhang Z, Wang T, Wang J,Zhou L, et al: BMP9 inhibits proliferation and metastasis of HER2-positive SK-BR-3 breast cancer cells through ERK1/2 and PI3K/AKT pathways. PLoS One 9: e96816, 2014.

14. Zardavas D, Fumagalli D and Loi S: Phosphatidylinositol 3-kinase/AKT/mammalian target of rapamycin pathway inhibition: A breakthrough in the management of luminal (ER+/HER2-) breast cancers? Curr Opin Oncol 24: 623-634, 2012.

15. Ciruelos Gil EM: Targeting the PI3K/AKT/mTOR pathway in estrogen receptor-positive breast cancer. Cancer Treat Rev 40: 862-871, 2014.

16. Grunt TW and Mariani GL: Novel approaches for molecular targeted therapy of breast cancer: Interfering with PI3K/AKT/mTOR signaling. Curr Cancer Drug Targets 13: 188-204, 2013.

17. Kang MH, Oh SC, Lee HJ, Kang HN, Kim JL, Kim JS and Yoo YA: Metastatic function of BMP-2 in gastric cancer cells: The role of PI3K/AKT, MAPK, the NF- $\mathrm{KB}$ pathway, and MMP-9 expression. Exp Cell Res 317: 1746-1762, 2011.

18. Shimizu T, Kayamori T, Murayama $\mathrm{C}$ and Miyamoto A: Bone morphogenetic protein (BMP)-4 and BMP-7 suppress granulosa cell apoptosis via different pathways: BMP-4 via PI3K/PDK-1/Akt and BMP-7 via PI3K/PDK-1/PKC. Biochem Biophys Res Commun 417: 869-873, 2012.

19. Wang K, Liu D, Zhu TJ, Tang ZG and Dai HY: BMP9 inhibit the proliferation, invasion and migration of MDA-MB-231 breast cancer cells through binding ALK2 receptor to activate BMPs/SMAD cell signaling pathway. Genomics and Applied Biology 35: 1569-1576, 2016.

20. Bolat Kucukzeybek B, Vedat Bayoglu I, Kucukzeybek Y, Alacacioglu A, Yigit S, Akder Sari A, Akyol $M$ and Oktay Tarhan M: The prognostic significance of cyclin D1 expression in patients with triple-negative breast cancer. J BUON 22: 947-952, 2017.

21. Khan S, Brougham CL, Ryan J, Sahrudin A, O'Neill G, Wall D, Curran C, Newell J, Kerin MJ and Dwyer RM: JmiR-379 regulates cyclin B1 expression and is decreased in breast cancer. PLoS One 8: e68753, 2013.

22. Guo X, Connick MC, Vanderhoof J, Ishak MA and Hartley RS: MicroRNA-16 modulates HuR regulation of Cyclin E1 in breast cancer cells. Int J Mol Sci 16: 7112-7132, 2015.

23. Wang J, Li M, Chen D, Nie J, Xi Y, Yang X, Chen Y and Yang Z: Expression of C-myc and $\beta$-catenin and their correlation in triple negative breast cancer. Minerva Med 108: 513-517, 2017.

24. Padala C, Tupurani MA, Puranam K, Gantala S, Shyamala N, Kondapalli MS, Gundapaneni KK, Mudigonda S, Galimudi RK, Kupsal K, et al: Synergistic effect of collagenase-1 (MMP1), stromelysin-1 (MMP3) and gelatinase-B (MMP9) gene polymorphisms in breast cancer. PLoS One 12: e0184448, 2017.

25. Li B, Yang Y, Jiang S, Ni B, Chen K and Jiang L: Adenovirusmediated overexpression of BMP-9 inhibits human osteosarcoma cell growth and migration through downregulation of the PI3K/AKT pathway. Int J Oncol 41: 1809-1819, 2012.

26. Pal I and Mandal M: PI3K and Akt as molecular targets for cancer therapy: Current clinical outcomes. Acta Pharmacol Sin 33: 1441-1458, 2012

27. Wang $\mathrm{Z}$ and Guo J: Mechanical induction of BMP-7 in osteocyte blocks glucocorticoid-induced apoptosis through PI3K/AKT/GSK3 $\beta$ pathway. Cell Biochem Biophys 67: 567-574, 2013. 
28. Zheng Y, Wang X, Wang H, Yan W, Zhang Q and Chang X: Bone morphogenetic protein 2 inhibits hepatocellular carcinoma growth and migration through downregulation of the PI3K/AKT pathway. Tumour Biol 35: 5189-5198, 2014.

29. Pancholi S, Lykkesfeldt A, Johnston SRD, Dowsett $M$ and Martin LA: The interaction of the ER with ERBB2 and PI3K results in elevated levels of AKT and p90RSK in tamoxifen-resistant MCF-7 cells. Breast Cancer Res 7 (Suppl 2): P2.08, 2005.

30. Lin M, Bi H, Yan Y, Huang W, Zhang G, Zhang G, Tang S, Liu Y, Zhang L, Ma J, et al: Parthenolide suppresses non-small cell lung cancer GLC-82 cells growth via B-Raf/MAPK/Erk pathway. Oncotarget 8: 23436-23447, 2017.

31. Duan L, Ye L, Wu R, Wang H, Li X, Li H, Yuan S, Zha H, Sun H, Zhang Y, et al: Inactivation of the phosphatidylinositol 3-kinase/Akt pathway is involved in BMP9-mediated tumor-suppressive effects in gastric cancer cells. J Cell Biochem 116: 1080-1089, 2015.

32. Ye L, Kynaston $\mathrm{H}$ and Jiang WG: Bone morphogenetic protein-9 induces apoptosis in prostate cancer cells, the role of prostate apoptosis response-4. Mol Cancer Res 6: 1594-1606, 2008.
33. Dai HY, Xia WY, Zhu TJ, Tang ZG and Wang K: BMP9 inhibits the proliferation of human lung adenocarcinoma A549 cells through BMPs/SMAD signaling pathway. TUMOR 35: 997-1005, 2015.

34. Liu P, Man Y, Wang Y and Bao Y: Mechanism of BMP9 promotes growth of osteosarcoma mediated by the Notch signaling pathway. Oncol Lett 11: 1367-1370, 2016.

35. Muñoz-Félix JM, Cuesta C, Perretta-Tejedor N, Subileau M, López-Hernández FJ, López-Novoa JM and Martínez-Salgado C: Identification of bone morphogenetic protein 9 (BMP9) as a novel profibrotic factor in vitro. Cell Signal 28: 1252-1261, 2016.

36. Yuan SX, Wang DX, Wu QX, Ren CM, Li Y, Chen QZ, Zeng YH, Shao Y, Yang JQ, Bai Y, et al: BMP9/p38 MAPK is essential for the antiproliferative effect of resveratrol on human colon cancer. Oncol Rep 35: 939-947, 2016.

37. Herrera B, van Dinther M, TenDijke P and Inman GJ: Autocrine bone morphogenetic protein-9 signals through activin receptor-like kinase-2/Smad1/Smad4 to promote ovarian cancer cell proliferation. Cancer Res 69: 9254-9262, 2009. 